\title{
2D Finite Element Method Simulation of Lateral Resonant Tunneling Devices
}

\author{
ZHI-AN SHAO, WOLFGANG POROD and CRAIG S. LENT \\ Department of Electrical Engineering, University of Notre Dame, Notre Dame, IN 46556
}

\begin{abstract}
Using the finite element method, we investigate device applications of lateral resonant tunneling structures which consist of a transmission channel with attached resonators. Such structure exhibits resonance-antiresonance transmission features which may be engineered to achieve desired device properties.

We show that the valley current can be reduced in such 2D lateral resonant tunneling devices, resulting in an improved current peak-to-valley ratio.
\end{abstract}

Keywords: lateral resonant tunneling, attached resonators, device modeling, valley current reduction, high current peak-to-valley ratio, finite element method

\section{INTRODUCTION}

It is believed that lateral resonant tunneling devices $[1,2]$ possess advantages over vertical ones. For example, the lateral device is more suitable for VLSI design and IC fabrication due to its planar structure. In this paper, based on our previous study of transmission resonance-antiresonances in quantum waveguide systems with resonantly-coupled cavities [3], we numerically investigate device applications of this transmission feature in single-barrier and double-barrier structures with attached stubs.

Recent studies have shown that transistor action can be achieved in various proposed stub tuner devices $[4,5]$. Subramaniam and co-workers studied the transmission characteristics of this device in the strongly-coupled case [6]. Even though complete transmission modulation is possible, the current peakto-valley ratio (PVR) of this device is low. Price further investigated resonance phenomena in this device [7] and pointed out that a weak coupling between the stub and the channel would lead to resonances due to the quasi-bound states of the stub. In previous study, we investigated the transmission properties of this structure and found that the resonance features predicted by Price actually are resonance-antiresonance features which correspond to zero-pole pairs in the complex-energy plane [3]. Here, we explore the applications of this transmission feature in weakly-coupled stub devices (a single-barrier structure with an inserted stub), and show that high current peak-io-valley ratios can be obtained.

In addition, we explore device applications of transmission zeros in double-barrier structures with an attached stub. For a double-barrier resonant tunneling (DBRT) device, the PVR is limited, even in the ballistic transport regime, since the transmission minimum has a finite value. Combining the transmission features of the DBRT devices and stub resonators, we shown that the valley current in such devices is reduced due to the zero-transmission feature of the stub. We also show that this device possesses a con- 
trollable lower peak voltage compared to lateral DBRT devices, where peak voltage refers to the voltage at which the maximum current is reached. We numerically model the above 2D lateral resonant tunneling structures, by the finite element method. In our treatment here, we have used hard wall potentials for simplicity A smooth potential may be more appropriate for a realistic device, however, previous studies have shown that this would not qualitatively alter the transmission features [8]. We first solve the 2D effective-mass Schrödinger equation to find the transmission probability $T(E, V)$ as a function of energy at different biases (or gate voltages), and then we calculate the $I-V$ characteristics by,

$$
I(V)=\frac{2 e}{h} \int_{0}^{\infty}[f(E)-f(E+e V)] T(E, V) d E,
$$

here $f(E)$ is the Fermi-Dirac distribution function. We choose a Fermi energy of $E_{f}=5 \mathrm{meV}$ in our calculation, which corresponds to a carrier density of $5.96 \times$ $10^{5}(1 / \mathrm{cm})$.

\section{SINGLE-BARRIER STRUCTURE WITH INSERTED STUB}

The structure of this device is schematically shown in the inset of figure 1 . The shaded area represents the potential barrier, which weakens the coupling between the stub and the channel. Through adjusting the length of the stub, (e.g. by a gate voltage), the quasi-bound state (or the resonance-antiresonance line shape) is altered, which modulates the transmission in the main channel. This feature of the transmission can be used to generate NDR regions with high PVR in the current versus gate voltage characteristics, which is superior to that of a strongly-coupled stub device [6].

The operation of this device depends on the variation of the quasi-bound states in the stub. When the stub length is short, the energy of the quasi-bound state is high, so the position of the transmission zero is far above the threshold of the first conduction band (the first propagating mode of the channel). Increas- ing the stub length lowers the quasi-bound state energy of the stub. Since there is a transmission maximum between the threshold and the first transmission zero [3], both positions of the transmission one and zero move toward the threshold. At a certain stub length, a maximum channel current is obtained. When the stub length is further increased, the transmission maximum falls below the threshold, and the current begins to drop. This generates a NDR region in the $I-V_{g}$ characteristics. After this drop, when the stub length is further increased, the second quasibound state of the stub comes into play and the above process repeats.

The analysis here is similar to DBRT except that the maximum current there corresponds to the minimum current here. This is not surprising since we know that the quasi-bound states in the stub resonator lead to transmission zeros instead of transmission ones, as in the case of DBRT. This analysis applies to both strongly- and weakly-coupled stub structures. However, because there is no sharp transmission variation for strongly-coupled stubs, the corresponding PVR is low. We will demonstrate this by an example.

Using the channel width, $W_{c h}$, as the basic unit of length, we choose the parameters for the structure shown in the inset of figure 1 as: $W_{s}=1.4 W_{c h}$. A gate voltage is used to control the length of the stub $L_{s}$, and a small bias $(0.7 \mathrm{mV}$, linear response regime $)$ is applied to the channel to generate a net current. Assuming the relationship between the stub length and the gate voltage is linear, we plot the current as a function of the stub length.

In figure 1, we show the current as a function of stub length at room temperature $T=300 \mathrm{~K}$ for various barrier heights $V_{h}$ (channel width is $5.0 \mathrm{~nm}$ and the corresponding threshold energy, $E_{t h}$, is $(0.22 \mathrm{eV})$. Part (a) shows the result for a strongly-coupled stub, $V_{h}=0 \mathrm{eV}$; and parts (b)-(d) show the results for weakly-coupled stubs with $V_{h}=0.05 \mathrm{eV}, 0.1 \mathrm{eV}$, and $0.15 \mathrm{eV}$, respectively. It is obvious that the NDR effect is obtained in each case. However, the current peak-to-valley ratios are different. The PVR's are high for the weakly-coupled cases and low for the strongly-coupled case. 


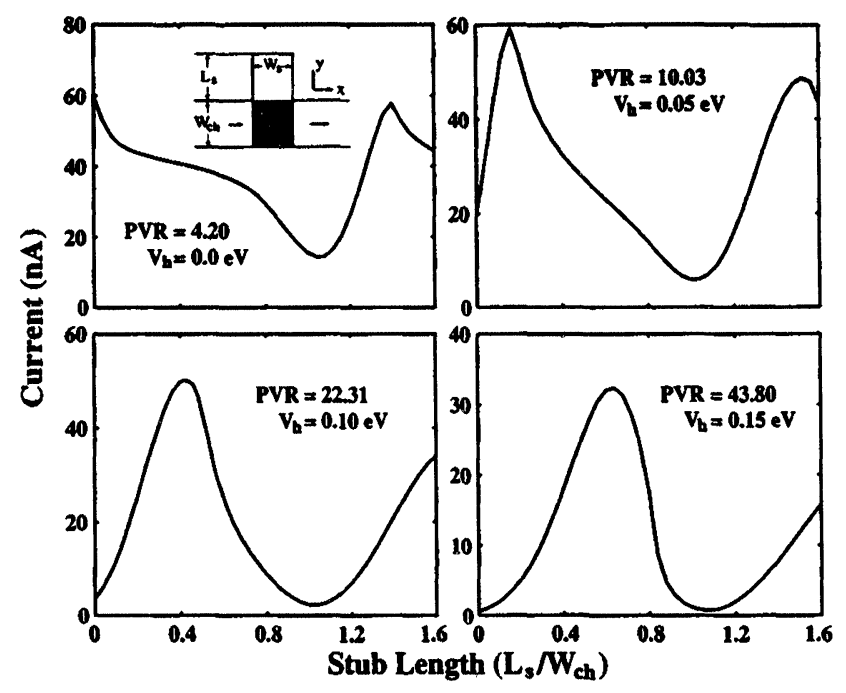

FIGURE 1 Current as a function of stub length for a single-barrier structure with an inserted stub at room temperature for various barrier height $V_{h}$. The device structure is schematically shown in the inset. (a) shows the result for a strongly-coupled stub; (b)-(d) show the results for the weakly-coupled stubs, respectively

\section{DOUBLE-BARRIER STRUCTURE WITH INSERTED STUB}

The structure of this device is schematically shown in the inset of figure 2. For a lateral DBRT device, the first transmission peak, $E_{p d b r}$, is determined by the energy of the first propagating mode of the channel, $E_{W_{c h}}=\hbar^{2} \pi^{2} /\left(2 m^{*} W_{c h}^{2}\right)$, and the first quasi-bound state energy of the corresponding 1D DBRT structure, $E_{1}=\hbar^{2} \pi^{2} /\left(2 m^{*} W_{s}^{2}\right)$, i.e., $E_{p d b r t}=E_{W_{c h}}+E_{1}$. Due to the inserted stub, for the structure shown in the inset of figure 2, the first transmission peak, $E_{p s t u b}$, is determined by the bound state energy of the stub in the y-direction, $E_{L}=\hbar^{2} \pi^{2} /\left(2 m^{*} L^{2}\right)$, and $E_{1}$, i.e., approximately $E_{p s t u b}=E_{L}+E_{1}$. Since $L$ is always larger than $W_{c h}$, the position of the first transmission peak for the structure shown in the inset of figure 2 is always lower than that of the corresponding lateral DBRT device, i.e., $E_{p s t u b}<E_{p d b r t}$.

In order to utilize the resonant nature of the first transmission peak and the transmission zero, the peak energy must be higher than the energy of the first propagating mode of the channel, i.e., $E_{p s t u b}>E_{W_{c h}}$.
We can achieve this condition by engineering the system parameters appropriately.

In resonant tunneling devices, the peak and valley currents are related to the transmission maximum and minimum, respectively. Since the transmission minimum is zero for the structure shown in the inset of figure 2 due to the inserted stub, its valley current is reduced compared to the lateral DBRT devices. Further, note that since $E_{p s t u b}<E_{p d b r t}$ and the position of $E_{\text {pstub }}$ can be engineered, a controllable low peak voltage can be obtained for the new device.

We use an example to illustrate our analysis. Using the channel width, $W_{c h}$, as the basic unit of length, we choose a set of parameters for the structure shown in the inset of figure 2: $L=2 W_{c h}, L_{b}=0.3 W_{c h}$, $W_{s}=0.8 W_{c h}$, and $V_{h}=0.45 \mathrm{eV}$. We show the $I-V$ characteristics of this structure at several temperatures from $T=100 \mathrm{~K}$ to $T=300 \mathrm{~K}$ (solid line) in figure 2 (channel width is $5.0 \mathrm{~nm}$ and the corresponding threshold energy, $E_{t h}$, is $0.22 \mathrm{eV}$ ). The results of the corresponding lateral DBRT are also shown (dashed line). It can be seen that the PVR of the new device $(\mathrm{PVR}=58.81,23.75,17.49,13.14)$ is improved over 


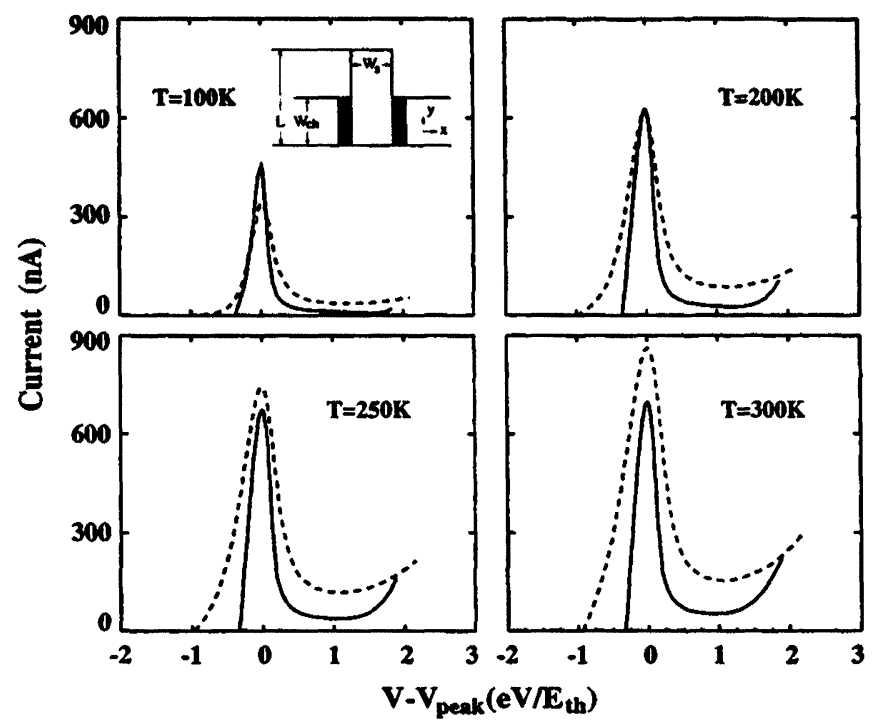

FIGURE $2 I-V$ characteristics of a double-barrier structure with an inserted stub (solid line) and the corresponding lateral DBRT structure (dashed line) at various temperatures. The device structure is schematically shown in the inset

the corresponding lateral DBRT device (PVR $=11.00$, $7.10,6.29,5.59)$. Note that the peak voltage, $V_{\text {peak }}$, is also low in this device, which is desirable for device applications.

In DBRT devices, a lower peak voltage is obtained when the well width is large $\left(E_{1}=\hbar^{2} \pi^{2} /\left(2 m^{*} W_{s}^{2}\right)\right)$. But, this also leads to a lower PVR since $E_{1}$ and $E_{2}$ are closer in this case. Therefore, there is a conflict between the two requirements in the device design. Here, by inserting a resonant cavity in the quantum well region of a DBRT structure, one may achieve both a higher current peak-to-valley ratio and a lower peak voltage.

\section{SUMMARY}

In summary, we have investigated single-barrier and double-barrier structures with attached stubs. Through engineering the transmission resonanceantiresonance feature, we find that high current peakto-valley ratios can be obtained. A controllable lower peak voltage is also achieved in the double-barrier device. These merits should offer better device performance.

\section{Acknowledgements}

This work is supported in part by ARPA/ONR and AFOSR.

\section{References}

[1] S. Y. Chou, D. R. Allee, R. F. W. Pease, and J. S. Harris, Jr, "Lateral Resonant Tunneling Transistors Employing FieldInduced Quantum Wells and Barriers," Proc. IEEE, vol. 79, pp. 1131-1139, 1991.

[2] J. N. Randall, A. C. Seabaugh, and J. H. Luscombe, "Fabrication of Lateral Resonant Tunneling Devices," J. Vac. Sci. Technol., vol. B10, pp. 2941-2944, 1992.

[3] W. Porod, Z. Shao, and C. S. Lent, "Transmission Resonances and Zeros in Quantum Waveguides with ResonantlyCoupled Cavities," Appl. Phys. Lett., vol. 61, pp. 1350-1352, 1992 and "Resonance-Antiresonance Line Shape for Transmission in Quantum Waveguides with Resonantly-Coupled Cavities," Phys. Rev. B, vol. 48, pp. 8495-8498, 1993 ; Z. Shao, W. Porod, and C. S. Lent, "Transmission Resonances and Zeros in Quantum Waveguide Systems with Attached Resonators," Phys. Rev. B, vol. 49, pp. 7453-7465, 1994.

[4] F. Sols, M. Macucci, U. Ravaioli, and K. Hess, "Theory for a Quantum Modulator Transistor," J. Appl. Phys., vol. 66, pp. 3892-3906, 1989.

[5] S. Datta, "Quantum Devices," Superlattices and Microstructures, vol. 6, pp. 83-93, 1989.

16] S. Subramaniam, S. Bandyopadhyay, and W. Porod, "Analysis of the Device Performance of Quantum Interference Transistors Utilizing Ultrasmall Semiconductor T Structures," J. Appl. Phys., vol. 68, pp. 4861-4870, 1990.

[7] P. J. Price, "Nanostructure Resonances," IEEE Trans. Electron. Dev., vol. 39, pp. 520-522, 1992.

[8] C. S. Lent and S. Sivaprakasam, "Quantum Electron Waveguides: Bends, Constrictions, and Cavities," Proc. SPIE, vol. 1284, pp. 31-38, 1990. 


\section{Biographies}

Zhi-an Shao is currently a Post-Doctoral Research Associate in the Department of Electrical Engineering at University of Notre Dame. His research interests include computational modeling of solid state electronics, semiconductor device simulation, and microelectronic material engineering. $\mathrm{He}$ is a member of Institute of Electrical and Electronics Engineers (IEEE) and American Institute of Physics.

Wolfgang Porod is Professor of Electrical Engineering at the University of Notre Dame. His research interests include computational solid state electronics and the physics of computation. He is a member of the APS and a senior member of the IEEE.

Craig S. Lent is currently Associate Professor of Electrical Engineering at the University of Notre Dame. His research interests include quantum computing, novel solid state devices, device modeling, and electromigration. $\mathrm{He}$ is a member of IEEE and APS. 

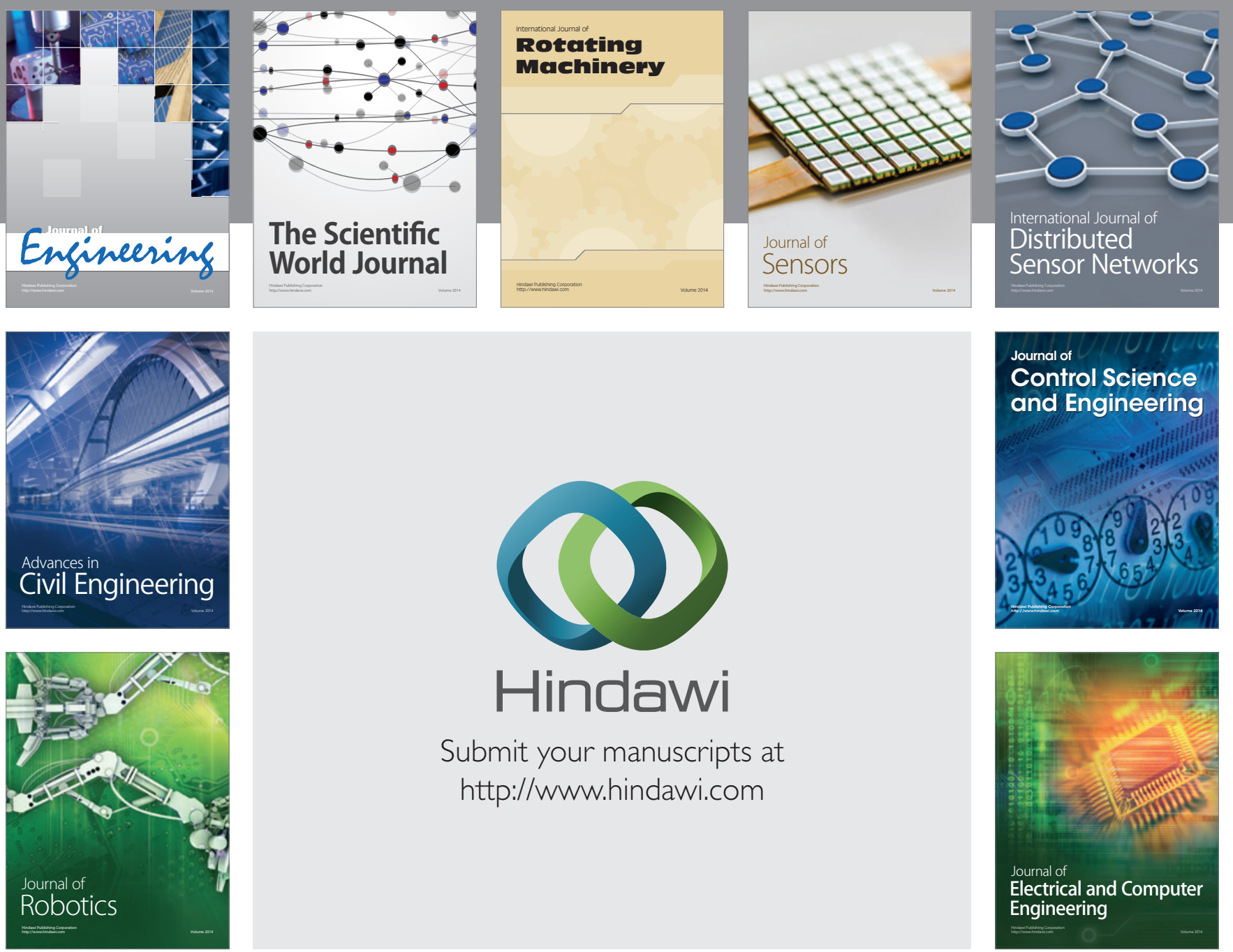

Submit your manuscripts at

http://www.hindawi.com
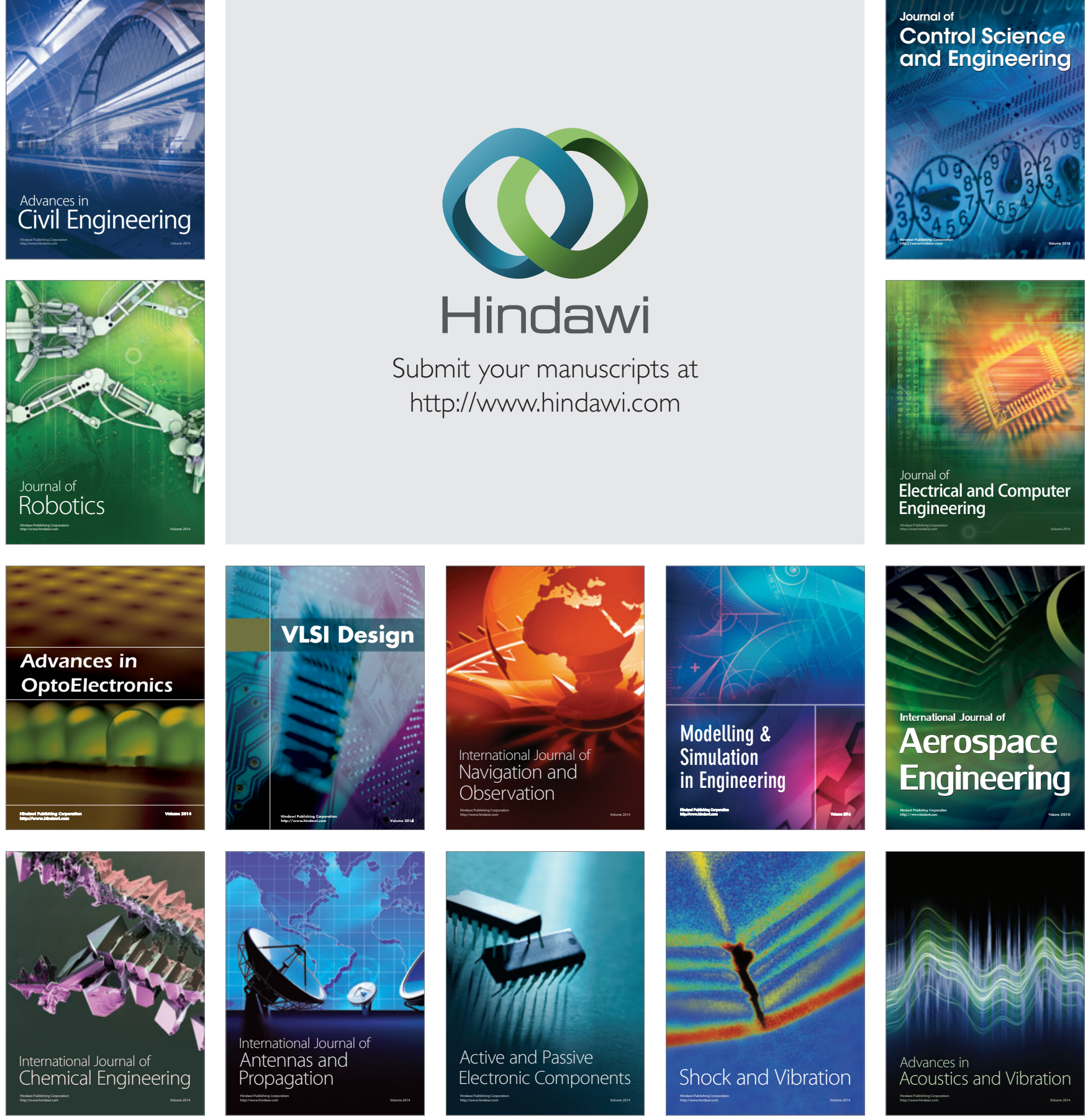